Article

\title{
State-of-Health Identification of Lithium-Ion Batteries Based on Nonlinear Frequency Response Analysis: First Steps with Machine Learning
}

\author{
Nina Harting ${ }^{1,2}$, René Schenkendorf ${ }^{1,2}$ (D), Nicolas Wolff ${ }^{1,2}$ and Ulrike Krewer ${ }^{1,2, *(1)}$ \\ 1 Institute of Energy and Process Systems Engineering, TU Braunschweig, Franz-Liszt-Straße 35, \\ 38106 Braunschweig, Germany; n.harting@tu-braunschweig.de (N.H.); \\ r.schenkendorf@tu-braunschweig.de (R.S.); n.wolff@tu-braunschweig.de (N.W.) \\ 2 Battery LabFactory Braunschweig, TU Braunschweig, Langer Kamp 19, 38106 Braunschweig, Germany \\ * Correspondence: u.krewer@tu-braunschweig.de; Tel.: +49-531-391-3030
}

Received: 27 April 2018; Accepted: 17 May 2018; Published: 19 May 2018

\begin{abstract}
In this study, we show an effective data-driven identification of the State-of-Health of Lithium-ion batteries by Nonlinear Frequency Response Analysis. A degradation model based on support vector regression is derived from highly informative Nonlinear Frequency Response Analysis data sets. First, an ageing test of a Lithium-ion battery at $25^{\circ} \mathrm{C}$ is presented and the impact of relevant ageing mechanisms on the nonlinear dynamics of the cells is analysed. A correlation measure is used to identify the most sensitive frequency range for ageing tests. Here, the mid-frequency range from $1 \mathrm{~Hz}$ to $100 \mathrm{~Hz}$ shows the strongest correlation to Lithium-ion battery degradation. The focus on the mid-frequency range leads to a dramatic reduction in measurement time of up to $92 \%$ compared to standard measurement protocols. Next, informative features are extracted and used to parametrise the support vector regression model for the State of Health degradation. The performance of the degradation model is validated with additional cells and validation data sets, respectively. We show that the degradation model accurately predicts the State of Health values. Validation data demonstrate the usefulness of the Nonlinear Frequency Response Analysis as an effective and fast State of Health identification method and as a versatile tool in the diagnosis of ageing of Lithium-ion batteries in general.
\end{abstract}

Keywords: lithium-ion batteries; state-of-health; nonlinear frequency response analysis; battery degradation; machine learning; support vector regression

\section{Introduction}

Lithium-ion batteries (LIBs) are currently the most widely used type of battery for electromotive applications and are seen as the most promising candidate for the realisation of a comprehensive electro-mobility. Current research focuses on the optimisation of safety as well as ageing diagnostics and lifetime predictions. The cell design, its operation and the environmental conditions all affect the ageing of a cell and therefore the lifespan of LIBs [1,2]. Various LIB degradation processes, which lead to a distinct decrease of the maximal usable capacity $C$ as well as an increase of the internal ohmic resistance $R$ of the LIB, can be distinguished [1-3]. In the field of e-mobility, for instance, the LIB degradation results in a loss of driving range per charge in electric vehicles (EVs) [2] and therefore in the deterioration of the coulombic efficiency $\eta$ of LIBs. Ageing processes occurring at the negative and the positive electrodes differ significantly and are usually differentiated in the literature [1]. Ageing processes in the electrolyte and the separator mostly result, respectively, from reactions with the electrodes and reactions at the electrode-electrolyte interface [1]. The most prominent ageing process 
at the negative electrode, the anode, is the formation of the solid electrolyte interface (SEI) [4] as well as Lithium plating $[5,6]$. At the positive electrode, the cathode, structural changes during cycling in combination with chemical decomposition reactions of the electrode material result in capacity decreases and therefore decreasing charging efficiency [1]. Furthermore, calendar and cyclic ageing of LIBs can be distinguished where both depend on environmental conditions [2,7]. Additionally, the State-of-Charge at which the battery is stored impacts calendar ageing [8]. Regarding cyclic ageing, the depth of discharge $(\mathrm{DoD})$ also impacts battery degradation processes [9]. Moreover, manufacturing aspects, i.e., variations in raw materials and assembly accuracy, complete the picture of relevant ageing factors. Following a cause-and-effect principle, we summarised the main factors influencing battery degradation and their interactions in an Ishikawa diagram; see Figure 1. In this work, we do not aim to identify and analyse dedicated ageing mechanisms but focus on higher-level LIB diagnostics tailored for battery management systems (BMSs).

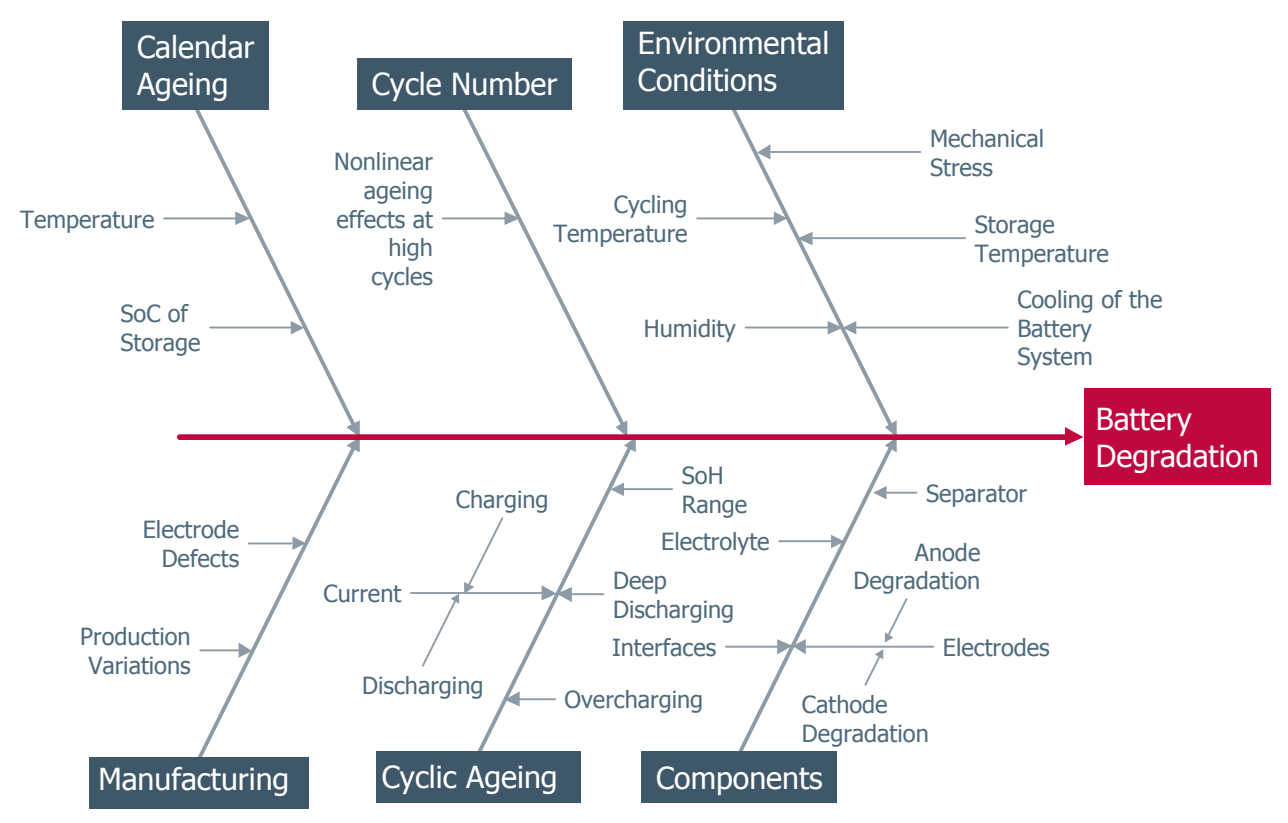

Figure 1. Ishikawa diagram of the various ageing factors leading to Lithium-ion battery degradation.

Reliable and precise state diagnosis of LIBs, e.g., State-of-Health (SoH), the State-of-Charge (SoC), and the characteristic remaining useful life (RUL) values, is therefore essential for reducing safety risks and estimating and extending the cycle life of battery systems used in EVs [8]. The accurate diagnosis of $\mathrm{SoH}$ is an indispensable part of effective battery health management for every BMS [10]. The SoH is mostly characterised by the maximum usable residual capacity $C$ or by the relative change in the internal ohmic resistance $R$ [11]. Typically, BMS uses such values as the capacity decrease and the power fade based on the increase of $R$ with regard to the nominal values $C_{0}$ and $R_{0}$ to quantify the SoH [12].

For a non-destructive determination of the SoH and the RUL under operating conditions or at rest, electrochemical in situ measurement methods are mandatory. Besides monitoring open-circuit-voltage $(\mathrm{OCV})$, the internal resistance $R$, determined via constant and pulsed current-voltage measurements, can be used to monitor the $\mathrm{SoH}[13,14]$. Other approaches use the internal resistance $R$ for the estimation of the SoH [15-17]. For the measurement of cell capacity, various in situ techniques are proposed in the literature, such as incremental capacity analysis (ICA) [18], differential voltage analysis (DVA) [19], combined IC-DV analysis techniques [20] as well as electrochemical impedance spectroscopy (EIS) [21]. Therefore, the use of ICA, DVA and EIS is suitable for SoH diagnosis as well as for the identification and differentiation of various ageing mechanisms [20,22]. These methods have specific advantages 
in SoH estimation. EIS has a shorter measurement time and can be used at particular frequencies for SoC. In contrast, ICA and DVA have the drawback of an extended test duration of approximately $10 \mathrm{~h}$. Furthermore, these methods are not suitable for specific cell states [21]. However, ICA and DVA are superior to EIS in terms of their universal, model-independent applications and low-cost hardware implementations as well as their easy calculation [20]. Nevertheless, LIBs have to be in a steady-state operating condition to apply the previously mentioned diagnostic techniques. Another disadvantage of EIS is that cell processes with a nonlinear current-voltage relation are only slightly excited in EIS due to the low excitation amplitude. Hence, only the linear system behaviour is observed, and relevant nonlinear cell information is not accessible for LIB diagnostics and SoH identification. In general, an efficient BMS benefits from a fast, cheap and accurate on-board SoH diagnosis of the state and the cycle life of LIBs [10,23].

Recently, a novel dynamic analysis method, the Nonlinear Frequency Response Analysis (NFRA), was established and applied to LIBs to investigate cell behaviour when it is excited with a nonlinear signal [24-26]. Thus, the effect of ageing mechanisms on the measured cell response is enhanced, and NFRA-based data might be more suitable for reliable SoH identification [24]. The application of NFRA for SoH estimation and RUL prediction is summarised in Figure 2 using a SWOT matrix to assess the NFRA concept as well as its application in LIB diagnostics and SoH identification. The strengths and opportunities of NFRA in LIB diagnostics look promising and motivate our experimental study. The weaknesses and threats seem to be manageable, in particular, as NFRA is a mature tool used in the state diagnosis of non-electrochemical systems. In 1985, NFRA was used for the first time to determine the SoC of lead-acid batteries [27]. In the field of fuel cells, at the stack and cell level, NFRA was successfully applied for system monitoring and analysis [28-30], but it was also used in more general kinetic studies [31,32].

\begin{tabular}{|c|c|c|}
\hline & Positive & Negative \\
\hline \multirow[b]{2}{*}{ 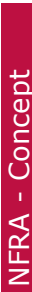 } & Strengths & Weaknesses \\
\hline & $\begin{array}{l}\text { - no need for steady-state analysis } \\
\text { - specific frequency range } \\
\text { - dependent on applied amplitudes } \\
\text { - high currents reveal nonlinear system } \\
\text { information }\end{array}$ & $\begin{array}{l}\text { - novel concept / fewer experiences } \\
\text { - nonintuitive } \\
\text { - no dedicated hardware / } \\
\text { measurement devices available } \\
\text { - challenging noise to signal ratio }\end{array}$ \\
\hline \multirow[b]{2}{*}{ 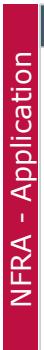 } & Opportunities & Threats \\
\hline & $\begin{array}{l}\text { - detailed LIB characterisation } \\
\text { - reveals dedicated reaction } \\
\text { mechanisms } \\
\text { - credible SoH identification } \\
\text { - precise fault detection \& identification } \\
\text { - online diagnosis under operating } \\
\text { conditions }\end{array}$ & $\begin{array}{l}\text { - safety margin issues because of high } \\
\text { currents } \\
\text { - unique identification of relevant LIB } \\
\text { characteristics fails } \\
\text { - no market acceptance }\end{array}$ \\
\hline
\end{tabular}

Figure 2. Strengths-Weakness-Opportunities-Threats (SWOT) analysis of the NFRA for LIB characterisation.

Over the last decade, data mining approaches have become popular for diagnosing and predicting the cell state of LIBs in BMSs. There are different approaches to unambiguously identify the complex and multi-scale ageing of LIBs and to predict cell state indicators. Common data-driven diagnostic algorithms use artificial neural networks as well as support vector machines (SVMs). Both methods have already been implemented for state identification and for determining the RUL of LIBs for simple test cases [33-35]. Since the accuracy of the data-driven processes depends critically on the data basis, 
one also attempts to increase the information content of the raw data with the help of first-principles models. Hybrid methods ideally combine the benefits of data mining with those of classical modelling principles [36,37]. Furthermore, they serve as the basis for data mining of electrochemical manipulated variables and measurements, such as current, voltage, internal resistance, maximum usable capacity and impedances $[21,38]$. To the authors' knowledge, diagnostic algorithms, which also take NFRA data for LIB diagnosis into account, are currently not available in the literature.

In this work, we analyse the applicability of NFRA as a diagnostic tool for LIBs and their SoH. Therefore, an ageing test of LIBs at $25^{\circ} \mathrm{C}$ is presented, and the impact of relevant ageing mechanisms on the nonlinear dynamics of the cells is explained in detail. Following a data mining strategy, an empirical degradation model is derived from informative NFRA data sets. First, highly diagnostic features are extracted and used to calibrate the degradation model for the NFR-driven SoH identification. Finally, the performance of the degradation model is validated with additional cells and validation data, respectively.

\section{Methods}

In this section, methods for SoH identification of LIBs are presented. First, NFRA as a dynamic ageing analysis method is introduced to gather informative data. Next, the Spearman rank correlation is used to determine the relevant frequencies related to LIB ageing and NFRA data, respectively. NFRA data limited to meaningful frequency ranges are processed to build the degradation model, which is implemented as a support vector regression (SVR) approach.

\subsection{Nonlinear Frequency Response Analysis (NFRA)}

With NFRA, a sinusoidal input signal with high current amplitudes $I_{A C}$ is applied to the system in a defined frequency range from $\mathrm{mHz}$ to $\mathrm{kHz}$. Changes in the output voltage $U_{A C}$ in the time domain are measured. By applying a Fast Fourier Transformation (FFT), the sinusoidal voltage output signal $U_{A C}$ is transferred from the time domain to the frequency domain. However, not only the voltage signal corresponding to input frequency $Y_{1}$, but also higher harmonic responses $Y_{n}$ with $n \geq 1$ are detected. Higher harmonic amplitudes of the responses $Y_{2}$ to $Y_{n}$ are observed at multiples of the fundamental frequency $f_{1}$ of the sinusoidal input signal:

$$
f_{n}=n \cdot f_{1}
$$

Even $Y_{2 n}$ and odd harmonics $Y_{2 n+1}$ have different characteristic responses. We examined these LIB characteristics in our previous work by investigating individual higher harmonic responses $Y_{n}$ as well as the corresponding sum $\sum_{i=2}^{n} Y_{i}$ over the frequency [24]. In general, the values for Nonlinear Frequency Responses (NFRs) correspond to the sum of nonlinearities $\sum_{i=2}^{5} Y_{i}$ of the investigated cell. The working principle of NFRA as well as the processing of the voltage output from the time in frequency domain using an FFT is illustrated in Figure 3.

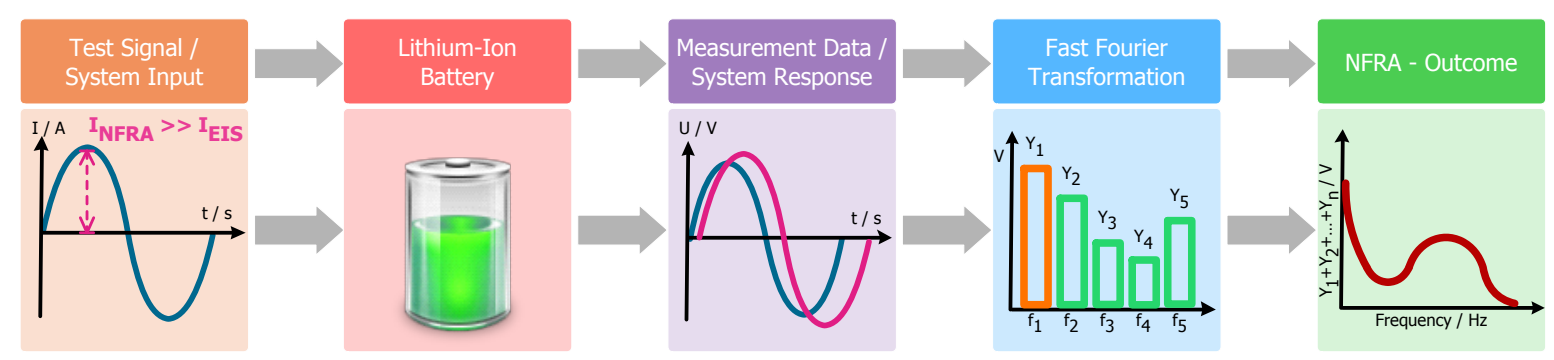

Figure 3. NFRA-scheme making use of higher-order harmonics. 


\subsection{SoH Degradation Model Based on Machine Learning}

In the literature, we can find various concepts for $\mathrm{SoH}$ degradation modelling which can be classified as first-principles, empirical, and hybrid models. First principles models translate electrochemical knowledge in equation systems but are generally difficult to derive. First, relevant processes have to be determined, associated model equations defined, and model parameters precisely identified. Thus, experimental data must be available to calibrate first-principles models. Alternatively, empirical models can be directly extracted from exhaustive data sets without any detailed knowledge of the underlying electrochemical processes. Please note that the quality of the empirical models depends critically on the available data and may fall short in extrapolation in the case of limited experimental data. Hybrid models, in turn, combine first-principles and data-driven concepts to use the benefits of both concepts; i.e., no perfect system understanding is necessary and missing data are compensated for with mechanistic rules. In this study, research is focused on empirical models and data mining techniques. Besides clustering and classification, regression concepts are frequently used for correlated data that are represented by governing equations for their input-output relationships. Complex neural networks and deep learning principles might be good candidates for data-driven SoH identification. The motivation for the present study, however, was to demonstrate the practical relevance of NFRA in SoH identification as an interesting concept for the next generation of BMSs. Thus, we have consciously chosen a simple data-processing work-flow: (1) frequencies for NFRA which are highly representative for cell ageing are identified via a correlation analysis; (2) informative features of the NFR data are calculated; (3) a sensitivity analysis reveals the most informative feature; and, (4) based on this feature, a SVR model is derived for SoH identification. The overall work flow is summarised in Figure 4 and described in detail below.

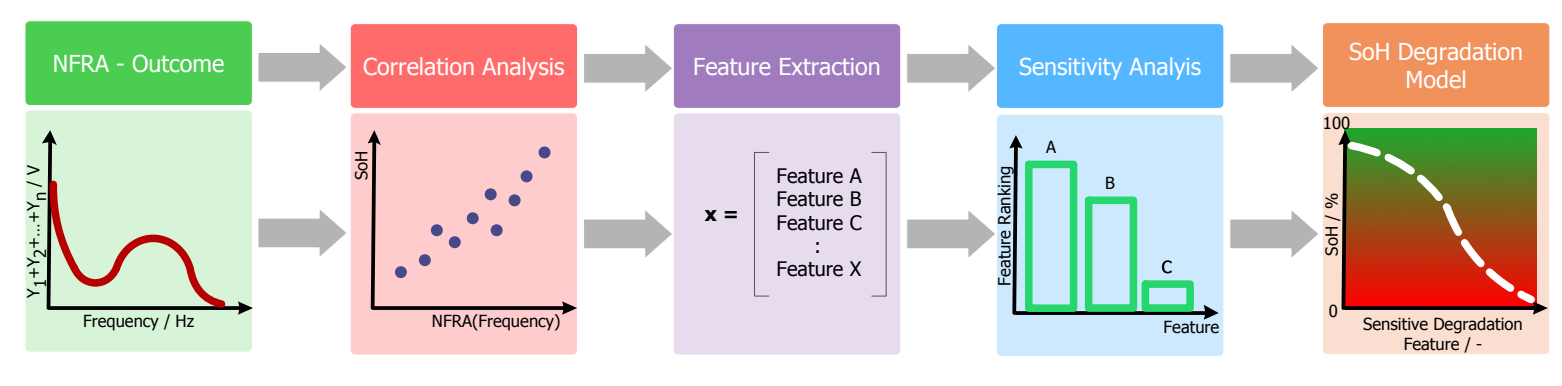

Figure 4. The overall data processing scheme for SoH identification.

\section{Correlation Analysis}

Correlation measures the strength of association between two variables, and its value varies between +1 and -1 for positive and negative relationships. A value of \pm 1 indicates a perfect degree of association between the two variables. As the correlation coefficient decreases towards 0 , the correlation between the two variables also declines.

In this study, Spearman rank [39] is used for testing the relationship between NFR and cycle number. Spearman rank correlation is a non-parametric test that measures the degree of association between two variables. Spearman rank correlation analysis does not need assumptions about the distribution of data and is the appropriate correlation analysis when the values of one variable are monotonically related to the other variable. Spearman rank correlation coefficient $\rho_{S}$ is calculated as:

$$
\rho_{S}=1-\frac{6 \sum d_{j}^{2}}{k\left(k^{2}-1\right)}
$$

where $d_{j}$ represents the difference between the ranks of two variables, and $k$ represents the number of observations. 


\subsection{Feature Extraction and Sensitivity Analysis}

To derive a data-driven SoH model, informative features are calculated from the NFRA data that cover the identified relevant frequency range. Various characteristics can be determined addressing geometrical, frequency, time-frequency, and statistical measures [40,41]. In this study, the geometrical features, $y$-axis intercept of the extrapolated NFR and the slope of the NFR spectra, are extracted for each ageing cycle. In detail, a simple linear regression analysis of the extracted NFR data in the sensitive frequency range is executed. Please note that the linear regression analysis is a low-cost and easy to implement approach, that is, an ideal method for the extraction of the relevant ageing features. In principle, additional features can be added, processed and translated to fewer but informative measures using principal component analysis and similar techniques [42,43]. Here, we directly select the most informative feature via a sensitivity study, that is, determining which feature changes the most with the cycle numbers. Only for this feature, an empirical SoH model is derived based on SVR.

\subsection{Support Vector Regression}

SVMs can be used for classification and regression $[44,45]$. In the case of regression, support vector machines are termed as support vector regression and is closely related to statistical learning. To build a regression model, a training data set $\mathcal{T}$ is required:

$$
\mathcal{T}=\{\vec{X}, \vec{Y}\}
$$

where $\vec{X}=\left\{x_{1}, \ldots, x_{K}\right\}$ are the input and $\vec{Y}=\left\{y_{1}, \ldots, y_{K}\right\}$ the output sets. The goal of the SVR is to approximate the data set via a regression model $F$ similar to:

$$
F(x)=\sum_{l=1}^{M} a_{l} x_{l}+b
$$

where the coefficients $b$ and $a_{l}, \forall l=1, \ldots, M$ are unknown and have to be identified using the training data set $\mathcal{T}$. Please note that the SVR was originally developed for linear regression problems but can be easily extended to nonlinear regression problems due to the so-called Kernel trick [46]. Moreover, empirical models based on SVR are robust to outliers, that is, data that are corrupted by large random errors or offsets. In addition, the SVR only considers those coefficients $a_{i}$ that are relevant and sets non-relevant coefficients to zero, which simplifies the model building and ensures well-posed identification problems in the case of limited data; that is, $K<M$. Thus, outliers and limited data have less impact on SVR models compared to ordinary regression techniques. For the technical details of SVR, the interested reader is referred to [46-48] and references therein. In the present study, frequency dependent NFR data sets during cycle ageing are included in the training data sets. Technically, we used the following SVR setting within the R statistical computing environment including the e1071 library: radial basis kernel, an epsilon value in the insensitive-loss function of 0.1 , and a C-constant of the regularisation term in the Lagrange formulation of 8.

\section{Case Study}

\subsection{Measurements and Cells}

LIBs in the pouch format with nickel-manganese-cobalt (NMC) as the cathode and graphite as the anode material were analysed in this ageing study. Electrode manufacturing (Figure 5a) and cell assembling (Figure 5b) were done by the Battery LabFactory Braunschweig (BLB) (Braunschweig, Germany), ensuring full control of the battery components. A solvent mixture of ethylene carbonate (EC) / diethyl carbonate (DEC) with a ratio of 3:7 with $1 \mathrm{M} \mathrm{LiPF}_{6}$ as the conducting salt and Celgard 2320 as the separator were used. The inactive compounds included are polyvinylidene fluoride as the binder and carbon black as the conducting additive. 


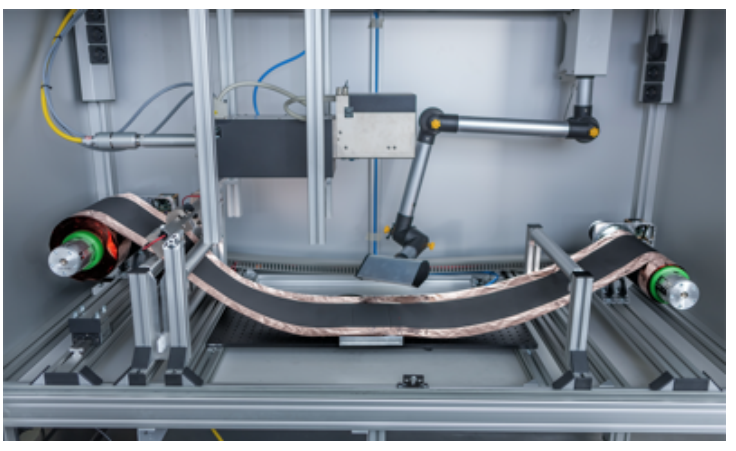

(a)

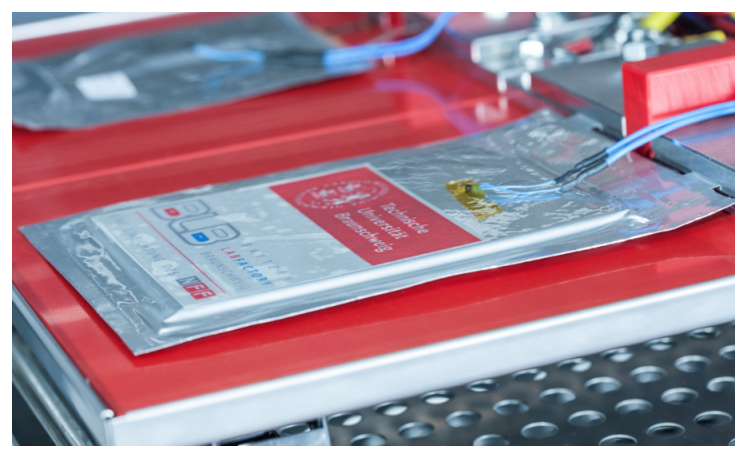

(b)

Figure 5. Electrode manufacturing and cell assembling at the Battery LabFactory Braunschweig (BLB). (a) BLB-Electrode production; (b) BLB-Pouch cell. (Picture credits: Hanno Keppel/Battery LabFactory, TU Braunschweig).

Cycle ageing experiments at $25^{\circ} \mathrm{C}$ were conducted by charging with $1 \mathrm{C}$ constant current $(\mathrm{CC})$ / constant voltage (CV) and discharging with $1 \mathrm{C} \mathrm{CC}$ in a potential window between $2.9 \mathrm{~V}$ and $4.2 \mathrm{~V}$. NFRA was measured with a Zahner Electrochemical Workstation (Zennium) in the galvanostatic mode in a temperature chamber also at a constant environmental temperature of $25^{\circ} \mathrm{C}$ prior to ageing and after each 50th cycle. Detailed measurement settings are given in [24], and cell specifications are summarised in Table 1.

Table 1. Performance and cell parameters of the investigated cells.

\begin{tabular}{lccc}
\hline Parameters & Symbol & Cell 1 & Cell 2 \\
\hline Initial capacity & $C_{0} / \mathrm{mAh}$ & 32.8 & 33.6 \\
Initial resistance & $R_{0} / \Omega$ & 0.21 & 0.29 \\
Anode thickness & $\delta_{a} \mu \mathrm{m}$ & 44 & 45 \\
Cathode thickness & $\delta_{c} \mu \mathrm{m}$ & 52 & 58 \\
Calendering degree anode & $\Pi_{a} / \%$ & 10 & 10 \\
Calendering degree cathode & $\Pi_{c} / \%$ & 10 & 0 \\
Mass fraction anode & $\zeta_{a} / \mathrm{wt} \%$ & 0.90 & 0.93 \\
Mass fraction cathode & $\zeta_{c} / \mathrm{wt} \%$ & 0.90 & 0.90 \\
Mass fraction inactive compounds anode & $\zeta_{a d d, a} / \mathrm{wt} \%$ & 0.10 & 0.07 \\
Mass fraction inactive compounds cathode & $\zeta_{a d d, c} / \mathrm{wt} \%$ & 0.10 & 0.10 \\
Porosity anode & $\varepsilon_{a} /-$ & 0.60 & 0.59 \\
Porosity cathode & $\varepsilon_{c} /-$ & 0.57 & 0.64 \\
Geometric surface area anode & $A_{g e o, a} / \mathrm{cm}^{2}$ & 30.25 & 30.25 \\
Geometric surface area cathode & $A_{g e o, c} / \mathrm{cm}^{2}$ & 30.25 & 30.25 \\
\hline
\end{tabular}

Typically, the SoH of an LIB is estimated by dividing the actual maximum residual capacity $C_{i}$ of the $i$-th cycles by the nominal capacity $C_{0}$ as in:

$$
\mathrm{SoH}_{i}=\frac{\mathrm{C}_{i}}{\mathrm{C}_{0}} \text {. }
$$

In the proposed case study, the $\mathrm{SoH}$ is calculated using highly informative NFR training data sets and is compared to the state-of-the art capacity based SoH estimation. This is motivated by the fact that we observed a monotonic correlation of NFR with capacity loss and increased resistance $R$ [24]. Thus, this indicates to evaluate directly the SoH via NFRA. 
The extrapolated NFR at the $y$-axis intercept is evaluated by linear regression analysis for all cycles $i, N F R_{\min , i}$ is used for this purpose and normalized with the extrapolated NFR at the $y$-axis intercept $N F R_{\min , 0}$ prior to ageing. The $S o H_{N F R}$ measure is defined as:

$$
\mathrm{SoH}_{N F R, i}=\frac{N F R_{\min , i}}{N F R_{\min , 0}} .
$$

Please note that with decreasing capacity $C_{0}$ and increasing internal resistance $R$, the sum of the higher harmonic amplitudes of the NFR increases for constant excitation amplitude $I_{A C}$. Thereby, it can be deduced that an increase of NFR correlates to a decrease of the SoH. The initial NFR value, NFR $\min , 0$ corresponds to a new cell $(\mathrm{SoH}=100 \%)$, and $N F R_{\min , i}$ correlates to a decreased $\mathrm{SoH}$ after $i$ cycles. Finally, the accuracy of the degradation model is calculated by comparing $\mathrm{SoH}_{S V M}$ to the $\mathrm{SoH}_{i}$, which is determined via standard capacity measurements during cycling.

\subsection{Results and Discussion}

Prior to NFR data analysis and the development of the degradation model, appropriate frequency ranges for the relevant processes had to be identified in the NFR spectrum of the ageing training data sets [24,26]. In Figure 6, NFR is shown over the frequency at specific cycle steps from 0 to 400 cycles, at each 50th cycle, respectively. Processes in the low-frequency range I from $0.02 \mathrm{~Hz}$ to $1 \mathrm{~Hz}$ can be attributed to diffusion processes in active material particles and processes in the mid-frequency range II from $1 \mathrm{~Hz}$ to approximately $300 \mathrm{~Hz}$ to electrochemical reactions. Processes in frequency range III, which show significantly lower NFR than the processes in ranges I and II, can most probably be attributed to ionic transport processes between and in the SEI and electrolyte. According to the literature, capacitive processes show almost minor or constant NFR in comparison to Faradaic processes with a Butler-Volmer kinetic. Furthermore, the separation of ranges I and II at approximately $1 \mathrm{~Hz}$ is distinct in the training data sets and, furthermore, is not affected by cell ageing. The identified process ranges with the corresponding time constants $\tau$ and frequencies $\omega$ are listed in Table 2. The correlation of processes to the frequency range in the NFR spectrum is supported by the corresponding impedance spectrum of the cell, which is shown in the inset of Figure 6 and discussed in detail in [24]. The NFR spectra in Figure 6 further illustrate that nonlinearities in the training data sets increase continuously with cycle ageing, particularly in frequency ranges I and II. In frequency range III, NFR spectra seem to be ageing-independent. However, without using data analysis methods, it is not possible to state with sufficient certainty an explicit ageing-to-NFR-correlation.

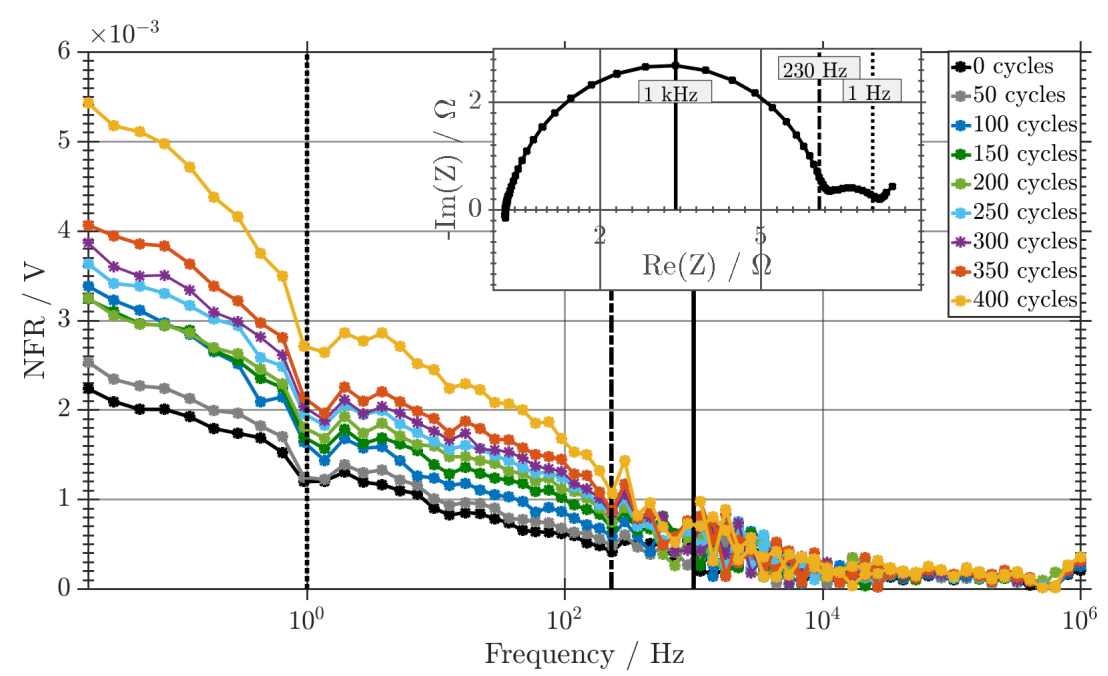

Figure 6. NFR spectra during cycle ageing at each 50th cycle, measured with $I_{A C}=1.6 \mathrm{C}$; the impedance spectrum initial to cycle ageing in the inset, is measured with $\mathrm{C} / 15 \mathrm{C}$. 
Table 2. Typical time constants and frequency ranges of processes identified for analysed LIB in the NFR spectra.

\begin{tabular}{ccc}
\hline$\tau / \mathbf{s}$ & $\omega$-Range $/ \mathbf{H z}$ & Process \\
\hline 50 to 1 & 0.02 to 1 & Solid diffusion \\
1 to 0.003 & 1 to 300 & Electrochemical reactions \\
0.003 to 0.0001 & 300 to 10,000 & Ionic transport processes at interfaces \\
\hline
\end{tabular}

Therefore, in the next step, a correlation analysis of the NFR and cycle number as a function of the analysed frequencies is calculated using the Spearman rank correlation measure. In Figure 7, the correlation coefficients for Spearman $\rho_{S}$ correlation is shown over frequency to identify the frequencies with relevant ageing information in the training data sets. Three characteristic frequency ranges with different ageing correlations are identified and can be distinctly separated at approximately $300 \mathrm{~Hz}$. For frequencies higher than $300 \mathrm{~Hz}$, range $C, \rho_{S}$ varies strongly and therefore NFR data extracted at those frequencies have no valid information about the ageing of the analysed LIB. For frequencies between $0.2 \mathrm{~Hz}$ and $300 \mathrm{~Hz}$, range $\mathrm{B}, \rho_{S}$ is exactly 1 , which indicates a perfect positive degree of association between NFR and cycle number. In the low frequency range $A, \rho_{S}$ differs from 1 ; $\rho_{S}$ extends from 0.93 to 0.98 , thereby indicating that NFR and the cycle number have a weaker positive relationship at these frequencies.

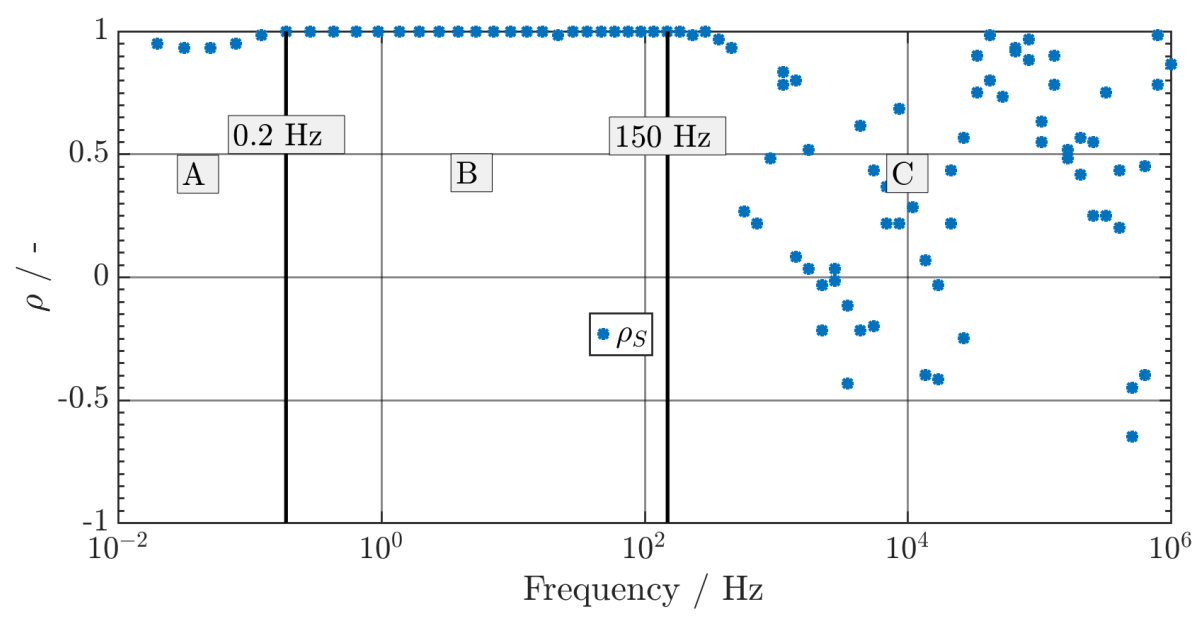

Figure 7. Correlation coefficient $\rho_{S}$ for estimating the correlation degree of the variables NFR and cycle number.

In the next step, the identified correlation ranges are interpreted by comparing correlation ranges A to $C$ with the identified processes in frequency ranges I to III, as shown in Table 3 . The most sensitive processes in the LIB are in the mid-frequency range from 0.2 to $300 \mathrm{~Hz}$ with a correlation coefficient of 1 for both types of correlation analysis methods. Thus, NFR data measured at these frequencies are highly suitable for LIB ageing quantification and the degradation model development. NFR data measured at frequencies lower than $0.2 \mathrm{~Hz}$ show a high correlation to ageing, as both correlation coefficients have values of $>0.95$. However, the data are not perfectly suitable in comparison with the higher frequency NFR data from $0.2 \mathrm{~Hz}$ to $150 \mathrm{~Hz}$. For frequencies higher than $150 \mathrm{~Hz}$, the NFR data have lower and strongly varying correlation coefficients for both correlation methods. Thus, NFR data at high frequencies have minor ageing sensitivities and are therefore not applicable for SoH quantification and not considered for use as training data for the degradation model. 
Table 3. Correlation ranges $\omega_{\text {Corr }}$, correlation coefficients and underlying LIB processes.

\begin{tabular}{ccc}
\hline$\omega_{\text {Corr }} / \mathrm{Hz}$ & Correlation Coefficient & Process \\
\hline A: $<0.2 \mathrm{~Hz}$ & $>0.95$ & Solid diffusion \\
B: 0.2 to 150 & 1 & Electrochemical reactions \\
C: $>150$ & Random & Ionic transport \\
\hline
\end{tabular}

The ageing-sensitive data identified for frequencies less than $150 \mathrm{~Hz}$ are extracted and shown over frequency in Figure 8.

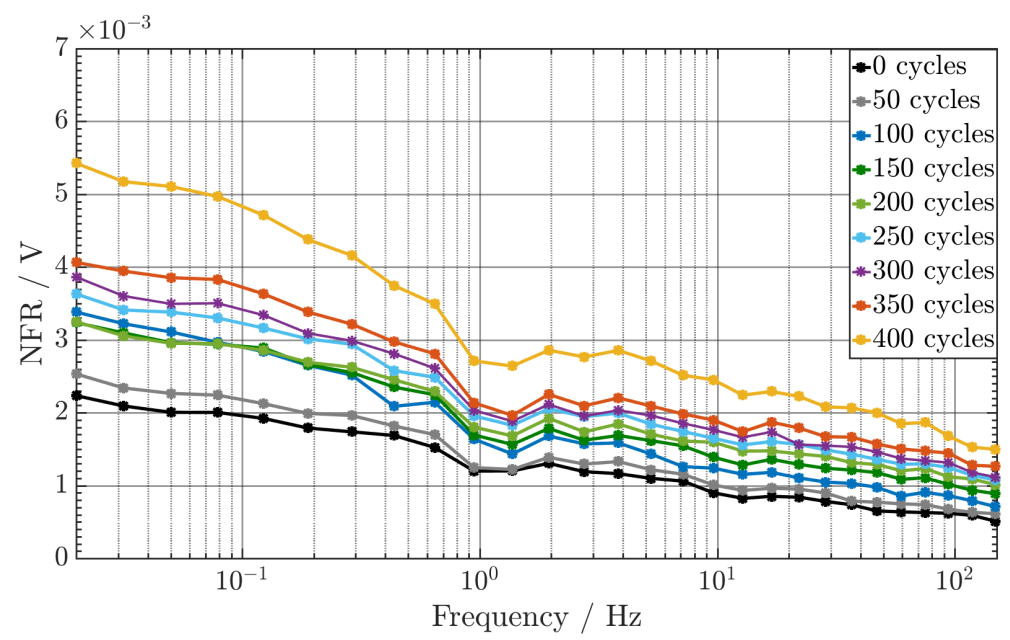

Figure 8. Ageing-sensitive features in the NFR spectra extracted from high correlating training data sets.

For calculating the degradation model using SVR, the geometrical features, $y$-axis intercept of the extrapolated NFR and the slope of the NFR spectra, are extracted for each ageing cycle. In Figure 9, the cycle specific feature values of extrapolated NFR at the $y$-axis intercept and the slope are plotted over the frequency of each cycle step. Apparently, extrapolated NFR at the $y$-axis intercept is more sensitive to ageing than the slope of the NFR spectra. Extrapolated NFR increases monotonically with the cycle number. The slope, however, deviates at cycles 100 and 150. Therefore, extrapolated NFR at the $y$-axis intercept is used for the parametrisation and training of the SVR degradation model. In Figure 10, the correlation of the SoH with the extracted degradation feature is shown. In the inset of Figure 10, the values of the degradation features extracted from the data (blue) as well as the calculated values by the SVR model (red) are shown in terms of the cycle number to illustrate the high accuracy of the degradation model as the measured and calculated values match perfectly. In the next step, the performance of the degradation model is tested with validation data sets and additional cells, which are non-identical to the initial test cells. NFR data sets identical and not identical to the training cells were applied to the degradation model, and the $\mathrm{SoH}$ was identified. The results of this validation are shown in Table 4. Moreover, the algorithm predicts the SoH of an identical cell with a high accuracy of $3 \%$ and the SoH of a non-identical cell with a high accuracy of $4 \%$. Thereby, it is shown that the NFR data recorded during cycle ageing are highly suitable for the quantification of the SoH of the cell for both identical and for non-identical cells. Please note that the battery cells used in the case study are manufactured by the Battery LabFactory Braunschweig and do not fulfill industrial standards, i.e., they may show substantial variations in their configurations that make a precise $\mathrm{SoH}$ identification difficult in general. The primary goal of this study was to provide the overall concepts and to demonstrate the proposed work-flow with first experimental results. Future work will focus on optimized and more sophisticated machine learning concepts with considerable higher accuracy, e.g., more informative features of NFRA data, empirical degradation models with memory (Bayesian concepts), and rigorous uncertainty analysis in SoH identification and $\mathrm{SoH}$ predictions. With NFRA, the measurement time 
can be reduced up to $92 \%$ compared to standard EIS protocols, as the ageing sensitivity of NFR data is sufficiently high in the mid-frequency range from $1 \mathrm{~Hz}$ to $100 \mathrm{~Hz}$ for the cells that are analysed in the presented study. Therefore, it is not necessary to analyse the overall frequency range from $\mathrm{mHz}$ to $\mathrm{kHz}$, which is typically done using dynamic measurement methods. Please also note that measuring the overall frequency range might be useful for SoH diagnosis of different cells and at different ageing conditions.

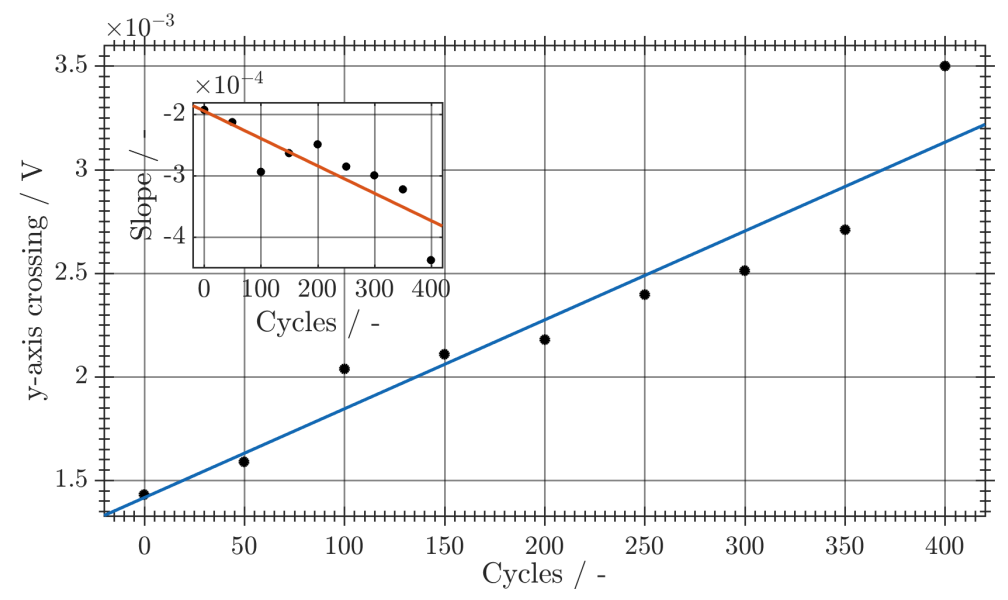

Figure 9. Ageing-sensitive NFR data extracted by correlation analysis.

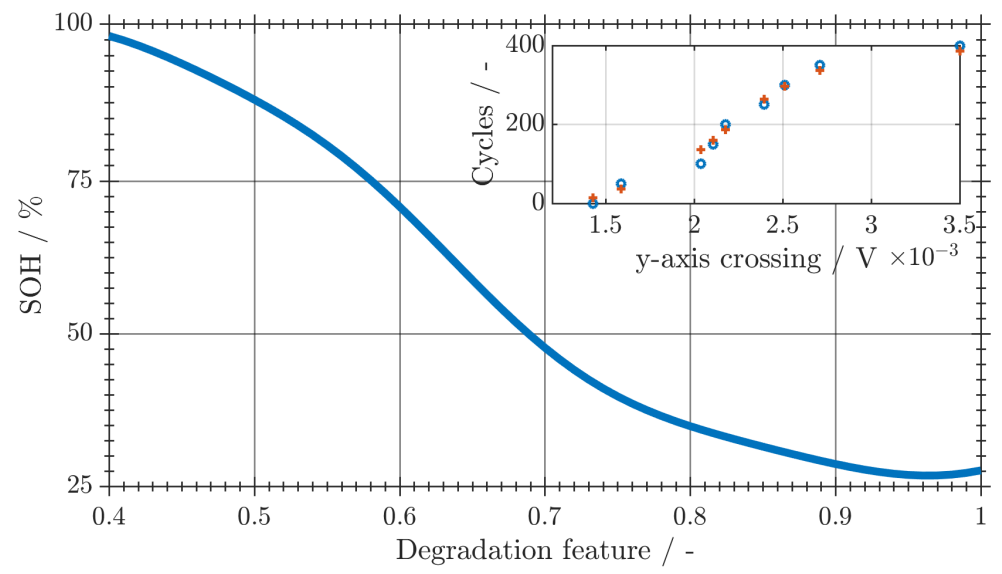

Figure 10. Degradation model for SoH estimation calculated by support vector regression (SVR) based on a highly ageing-sensitive data feature and in the inlet, the degradation feature values extracted from the data (blue) as well as the calculated values by the SVR model (red).

Table 4. Validation of the degradation model using identical and non-identical cells at different SoHs.

\begin{tabular}{ccccc}
\hline Cell & Type & SoH $_{i}$ & SoH $_{S V M}$ & Accuracy/\% \\
\hline B & identical & 28 & 29 & 3 \\
C & identical & 63 & 66 & 4 \\
D & non-identical & 100 & 96 & 4 \\
\hline
\end{tabular}

\section{Conclusions}

In this study, we demonstrated the effective data-driven identification of $\mathrm{SoH}$ by using the novel dynamic analysis method NFRA. First, an accelerated ageing test of an LIB at $25^{\circ} \mathrm{C}$ was executed and a dynamic analysis with NFRA performed in defined cycle steps at constant environmental and measurement conditions. The nonlinear dynamics of the cell changed regarding the ageing protocol 
with proceeding cycle numbers. The NFR increased monotonically with cycling, but the qualitative progress of the NFR spectra remained unaffected. The most likely ageing effects at the applied cycling current and temperature are electrolyte degradation and the growth of the SEI, respectively. Such ageing effects include capacity loss and increased cell impedance, which lead to higher NFR values, as observed in this ageing study.

For identifying the most sensitive frequency range for ageing tests, a correlation analysis was performed. Here, the mid-frequency range from $0.2 \mathrm{~Hz}$ to $150 \mathrm{~Hz}$ shows the strongest correlation with LIB degradation. After extraction of extrapolated NFR at the $y$-axis intercept and the slope as informative features for LIB ageing, the extrapolated NFR values at the $y$-axis intercept were used to parametrise the SVR model for SoH degradation, as it shows a higher correlation to NFR ageing data. By using additional cells and data sets, the degradation model was validated and tested. It was shown that the degradation model can predict the SoH values with high accuracy.

Taken together, these results demonstrate the usefulness of NFRA as an effective and fast SoH identification method as well as a versatile tool in the ageing diagnosis of LIBs in general. Furthermore, as the correlation coefficients indicate a perfect correlation of NFR to ageing for all frequencies between $0.2 \mathrm{~Hz}$ and $150 \mathrm{~Hz}$, we suggest a reduction of the measurement range from $1 \mathrm{~Hz}$ to $100 \mathrm{~Hz}$ for the analysed cells, which leads to a dramatic reduction in measurement time of up to $92 \%$ compared to standard measurement protocols. Future work will take the evaluation of NFRA data sets of commercial cells and the impact of changes in the measurement scenarios, such as temperature variations, as well as ageing conditions on the SoH identification algorithm into account. Furthermore, we presently work on the effect of temperature distribution within cells on NFRA; this is a first step to account the more complex behaviour of battery packs, which show temperature and property distributions.

Author Contributions: N.H. and R.S. wrote the manuscript; N.H. conceived, designed and performed the experiments; R.S. analysed the data with SVR; N.W. contributed to the analysis of results; U.K. contributed to the analysis of results, provided feedback to the content and participated in writing the manuscript.

Acknowledgments: The IGF project 8415 N/1 of the research association for Drive Technology (FVA e.V.) was funded by the German Federation of Industrial Research Associations (AiF) within the program Industrial Collective Research and Development (IGF) of the Federal Ministry of Economics and Technology (BMWi). Furthermore, we appreciate the support of the Battery LabFactory Braunschweig, notably the Institute for Particle Technology, for manufacturing the electrodes.

Conflicts of Interest: The authors declare no conflict of interest. The founding sponsors had no role in the design of the study; in the collection, analyses or interpretation of data; in the writing of the manuscript; and in the decision to publish the results.

\section{References}

1. Vetter, J.; Novak, P.; Wagner, M.; Veit, C. Ageing Mechanisms in Lithium-Ion Batteries. J. Power Sources 2005, 147, 269-281, doi:10.1016/j.jpowsour.2005.01.006.

2. Barré, A.; Deguilhem, B.; Grolleau, S.; Gérard, M.; Suard, F.; Riu, D. A review on Lithium-Ion Battery Ageing Mechanisms and Estimations for Automotive Applications. J. Power Sources 2013, 241, 680-689, doi:10.1016/j.jpowsour.2013.05.040.

3. Agubra, V.; Fergus, J. Lithium ion battery anode aging mechanisms. Materials 2013, 6, 1310-1325, doi:10.3390/ma6041310.

4. Verma, P.; Maire, P.; Novák, P. A Review of the Features and Analyses of the Solid Electrolyte Interphase in Li-Ion Batteries. Electrochim. Acta 2010, 55, 6332-6341, doi:10.1016/j.electacta.2010.05.072.

5. Petzl, M.; Kasper, M.; Danzer, M.A. Lithium Plating in a Commercial Lithium-Ion Battery-A LowTemperature Aging Study. J. Power Sources 2014, 275, 799-807, doi:10.1016/j.jpowsour.2014.11.065.

6. Waldmann, T.; Hogg, B.I.; Wohlfahrt-Mehrens, M. Li plating as unwanted side reaction in commercial Li-ion cells-A review. J. Power Sources 2018, 384, 107-124, doi:10.1016/j.jpowsour.2018.02.063.

7. Fleischhammer, M.; Waldmann, T.; Bisle, G.; Hogg, B.I.; Wohlfahrt-Mehrens, M. Interaction of Cyclic Ageing at High-Rate and Low Temperatures and Safety in Lithium-Ion Batteries. J. Power Sources 2015, 274, 432-439, doi:10.1016/j.jpowsour.2014.08.135. 
8. Wu, C.; Zhu, C.; Ge, Y.; Zhao, Y. A Review on Fault Mechanism and Diagnosis Approach for Li-Ion Batteries. J. Nanomater. 2015, 2015, 8, doi:10.1155/2015/631263.

9. Lewerenz, M.; Marongiu, A.; Warnecke, A.; Sauer, D.U. Differential voltage analysis as a tool for analyzing inhomogeneous aging: A case study for $\mathrm{LiFePO}_{4} \mid$ Graphite cylindrical cells. J. Power Sources 2017, 368, 57-67, doi:10.1016/j.jpowsour.2017.09.059.

10. Rezvanizaniani, S.M.; Liu, Z.; Chen, Y.; Lee, J. Review and recent advances in battery health monitoring and prognostics technologies for electric vehicle (EV) safety and mobility. J. Power Sources 2014, 256, 110-124, doi:10.1016/j.jpowsour.2014.01.085.

11. Broussely, M.; Biensan, P.; Bonhomme, F.; Blanchard, P.; Herreyre, S.; Nechev, K.; Staniewicz, R. Main Aging Mechanisms in Li Ion Batteries. J. Power Sources 2005, 146, 90-96, doi:10.1016/j.jpowsour.2005.03.172.

12. Zhou, Y.; Huang, M.; Chen, Y.; Tao, Y. A novel health indicator for on-line lithium-ion batteries remaining useful life prediction. J. Power Sources 2016, 321, 1-10, doi:10.1016/j.jpowsour.2016.04.119.

13. Liu, Q.; Du, C.; Shen, B.; Zuo, P.; Cheng, X.; Ma, Y.; Yin, G.; Gao, Y. Understanding undesirable anode lithium plating issues in lithium-ion batteries. RSC Adv. 2016, 6, 88683-88700, doi:10.1039/C6RA19482F.

14. Schindler, S.; Bauer, M.; Petzl, M.; Danzer, M.A. Voltage relaxation and impedance spectroscopy as in-operando methods for the detection of lithium plating on graphitic anodes in commercial lithium-ion cells. J. Power Sources 2016, 304, 170-180, doi:10.1016/j.jpowsour.2015.11.044.

15. Remmlinger, J.; Buchholz, M. State-of-Health Monitoring of Lithium-Ion Batteries in Electric Vehicles by on-board Internal Resistance Estimation. J. Power Sources 2011, 196, 5357-5363, doi:10.1016/j.jpowsour.2010.08.035.

16. Sepasi, S.; Ghorbani, R.; Liaw, B.Y. Inline state of health estimation of lithium-ion batteries using state of charge calculation. J. Power Sources 2015, 299, 246-254, doi:10.1016/j.jpowsour.2015.08.091.

17. Wang, Y.; Pan, R.; Yang, D.; Tang, X.; Chen, Z. Remaining Useful Life Prediction of Lithium-ion Battery Based on Discrete Wavelet Transform. Energy Procedia 2017, 105, 2053-2058, doi:10.1016/j.egypro.2017.03.582.

18. Dubarry, M.; Svoboda, V.; Hwu, R.; Yann Liaw, B. Incremental Capacity Analysis and Close-to-Equilibrium OCV Measurements to Quantify Capacity Fade in Commercial Rechargeable Lithium Batteries. Electrochem. Solid-State Lett. 2006, 9, A454-A457, doi:10.1149/1.2221767.

19. Waldmann, T.; Iturrondobeitia, A.; Kasper, M.; Ghanbari, N.; Aguesse, F.; Bekaert, E.; Daniel, L.; Genies, S.; Gordon, I.J.; Löble, M.W.; et al. Review-Post-Mortem Analysis of Aged Lithium-Ion Batteries: Disassembly Methodology and Physico-Chemical Analysis Techniques. J. Electrochem. Soc. 2016, 163, A2149-A2164, doi:10.1149/2.1211609jes.

20. Pastor-Fernández, C.; Uddin, K.; Chouchelamane, G.H.; Widanage, W.D.; Marco, J. A Comparison between Electrochemical Impedance Spectroscopy and Incremental Capacity-Differential Voltage as Li-ion Diagnostic Techniques to Identify and Quantify the Effects of Degradation Modes within Battery Management Systems. J. Power Sources 2017, 360, 301-318, doi:10.1016/j.jpowsour.2017.03.042.

21. Hung, M.H.; Lin, C.H.; Lee, L.C.; Wang, C.M. State-of-Charge and State-of-Health Estimation for Lithium-Ion Batteries based on Dynamic Impedance Technique. J. Power Sources 2014, 268, 861-873, doi:10.1016/j.jpowsour.2014.06.083.

22. Osaka, T.; Mukoyama, D.; Nara, H. Review_Development of Diagnostic Process for Commercially Available Batteries, Especially Lithium Ion Battery, by Electrochemical Impedance Spectroscopy. J. Electrochem. Soc. 2015, 162, A2529-A2537, doi:10.1149/2.0141514jes.

23. Lu, L.; Han, X.; Li, J.; Hua, J.; Ouyang, M. A Review on the Key Issues for Lithium-Ion Battery Management in Electric Vehicles. J. Power Sources 2013, 226, 272-288, doi:10.1016/j.jpowsour.2012.10.060.

24. Harting, N.; Wolff, N.; Röder, F.; Krewer, U. Nonlinear Frequency Response Analysis (NFRA) of Lithium-Ion Batteries. Electrochim. Acta 2017, doi:10.1016/j.electacta.2017.04.037.

25. Murbach, M.D.; Schwartz, D.T. Extending Newman's Pseudo-Two-Dimensional Lithium-Ion Battery Impedance Simulation Approach to Include the Nonlinear Harmonic Response. J. Electrochem. Soc. 2017, 164, E3311-E3320, doi:10.1149/2.0301711jes.

26. Wolff, N.; Harting, N.; Heinrich, M.; Röder, F.; Krewer, U. Nonlinear Frequency Response Analysis on Lithium-Ion Batteries: Pseudo-Two-Dimensional Porous Electrode Model Based Investigation. Electrochim. Acta 2017, 260, 614-622, doi:10.1016/j.electacta.2017.12.097.

27. Okazaki, S. Second-Order Harmonic in the Current Response to Sinusoidal Perturbation Voltage for Lead-Acid Battery. J. Electrochem. Soc. 1985, 132, 1516-1520, doi:10.1149/1.2114157. 
28. Ramschak, E.; Peinecke, V.; Prenninger, P.; Schaffer, T.; Baumgartner, W.; Hacker, V. Online Stack Monitoring Tool for Dynamically and Stationary Operated Fuel Cell Systems. Fuel Cells Bull. 2006, 2006, 12-15, doi:10.1016/S1464-2859(06)71207-X.

29. Ramschak, E.; Peinecke, V.; Prenninger, P.; Schaffer, T.; Hacker, V. Detection of Fuel Cell Critical Status by Stack Voltage Analysis. J. Power Sources 2006, 157, 837-840, doi:10.1016/j.jpowsour.2006.01.009.

30. Mao, Q.; Krewer, U. Sensing Methanol Concentration in Direct Methanol Fuel Cell with Total Harmonic Distortion: Theory and Application. Electrochim. Acta 2012, 68, 60-68, doi:10.1016/j.electacta.2012.02.018.

31. Mao, Q.; Krewer, U. Total Harmonic Distortion Analysis of Oxygen Reduction Reaction in Proton Exchange membrane fuel Cells. Electrochim. Acta 2013, 103, 188-198, doi:10.1016/j.electacta.2013.03.194.

32. Vidakovic, T.R.; Panic, V.V. Nonlinear Frequency Response Analysis of the Ferrocyanide Oxidation Kinetics. Part I. A Theoretical Analysis. J. Phys. Chem. C 2011, 115, 17341-17351.

33. Sbarufatti, C.; Corbetta, M.; Giglio, M.; Cadini, F. Adaptive prognosis of lithium-ion batteries based on the combination of particle filters and radial basis function neural networks. J. Power Sources 2017, 344, 128-140, doi:10.1016/j.jpowsour.2017.01.105.

34. Yang, D.; Wang, Y.; Pan, R.; Chen, R.; Chen, Z. A neural network based state-of-health estimation of lithium-ion battery in electric vehicles. Energy Procedia 2016, 105, 2059-2064, doi:10.1016/j.egypro.2017.03.583.

35. Dong, H.; Jin, X.; Lou, Y.; Wang, C. Lithium-ion battery state of health monitoring and remaining useful life prediction based on support vector regression-particle filter. J. Power Sources 2014, 271, 114-123, doi:10.1016/j.jpowsour.2014.07.176.

36. Panchal, S.; Mcgrory, J.; Kong, J.; Fraser, R.; Fowler, M.; Dincer, I.; Agelin-Chaab, M. Cycling degradation testing and analysis of a LiFePO4 battery at actual conditions. Int. J. Energy Res. 2017, 41, 2565-2575.

37. Ye, M.; Guo, H.; Xiong, R.; Yang, R. Model-based State-of-charge Estimation Approach of the Lithium-ion Battery Using an Improved Adaptive Particle Filter. Energy Procedia 2016, 103, 394-399, doi:10.1016/j.egypro.2016.11.305.

38. Wang, Y.; Yang, D.; Zhang, X.; Chen, Z. Probability based remaining capacity estimation using data-driven and neural network model. J. Power Sources 2016, 315, 199-208, doi:10.1016/j.jpowsour.2016.03.054.

39. Gibbons, J.D.; Chakraborti, S. Nonparametric statistical inference. In International Encyclopedia of Statistical Science; Springer: Berlin/Heidelberg, Germany, 2003; Volume 15, p. 645, doi:10.2307/1267003.

40. Jovic, A.; Brkic, K.; Bogunovic, N. A review of feature selection methods with applications. In Proceedings of the 2015 38th International Convention on Information and Communication Technology, Electronics and Microelectronics (MIPRO), Opatija, Croatia, 25-29 May 2015; pp. 1200-1205, doi:10.1109/MIPRO.2015.7160458.

41. Dy, J.G.; Brodley, C.E. Feature Selection for Unsupervised Learning. J. Mach. Learn. Res. 2004, 5, 845-889.

42. Fu, T.C. A review on time series data mining. Eng. Appl. Artif. Intell. 2011, 24, 164-181.

43. Severson, K.; Chaiwatanodom, P.; Braatz, R.D. Perspectives on process monitoring of industrial systems. In Proceedings of the 9th IFAC Symposium on Fault Detection, Supervision and Safety for Technical Processes (SAFEPROCESS), Paris, France, 2-4 September 2015; pp. 931-939.

44. Smola, A.J.; Sc Olkopf, B. A tutorial on support vector regression. Stat. Comput. 2004, 14, 199-222, doi:10.1023/B:STCO.0000035301.49549.88.

45. Zhou, J.; Liu, D.; Peng, Y.; Peng, X. Dynamic battery remaining useful life estimation: An on-line data-driven approach. In Proceedings of the 2012 IEEE International Instrumentation and Measurement Technology Conference (I2MTC), Graz, Austria, 13-16 May 2012; pp. 2196-2199, doi:10.1109/I2MTC.2012.6229280.

46. Schölkopf, B.; Smola, A.J. Learning with Kernels: Support Vector Machines, Regularization, Optimization, and Beyond; MIT Press: Cambridge, MA, USA, 2002.

47. Cortes, C.; Vapnik, V. Support-vector networks. Mach. Learn. 1995, 20, 273-297.

48. Liao, L.; Köttig, F. Review of Hybrid Prognostics Approaches for Remaining Useful Life Prediction of Engineered Systems, and an Application to Battery Life Prediction. IEEE Trans. Reliab. 2014, 63, 191-207.

(C) 2018 by the authors. Licensee MDPI, Basel, Switzerland. This article is an open access article distributed under the terms and conditions of the Creative Commons Attribution (CC BY) license (http:/ / creativecommons.org/licenses/by/4.0/). 